\title{
Isolation Purification and Structure Elucidation of Antibacterial Active Compounds from Freshwater Cyanobacterium Lyngbya sp.
}

\author{
Wijesekara W.A.M.A., Manage P.M.* \\ Center for Water Quality \& Algae Research, Department of Zoology, \\ University of Sri Jayewardenepura, Sri Lanka. \\ *pathmalal@sjp.ac.lk
}

\begin{abstract}
Cyanobacteria are considered as a rich source of bioactive compounds as they are able to produce a great variety of secondary metabolites characterized by a broad spectrum of biological activities including antiviral, antibacterial, antifungal, and anticancer properties. In the present study, freshwater cyanobacterium Lyngbya sp. was isolated from the Sennayaka samudraya and methanol crude extract was selected based on the previous antibacterial study (Agar disc diffusion method and Broth Microdialution method (Minimum Inhibition Concentration) to isolate and purify antibacterial active compounds. The antimicrobial activity of the methanol crude extract of Lynbya sp. was tested against two human pathogenic Gram-positive bacteria (Bacillus sp., Staphylococcus aureus-ATCC 2593), two human pathogenic Gram-negative bacteria (Escherichia coli-ATCC 25922, 2785 and Salmonella typhi) using bioautochromatography. Ciprofloxacin $(5 \mu \mathrm{g} / \mathrm{disc})$ was used as a positive control whereas DMSO (100\%) used as negative control. The antioxidant property in the crude extract was evaluated using DPPH (2,2-diphenyl-1-picrylhydrazyl) scavenging test (Inhibitory Concentration, $\mathrm{IC}_{50}=120.042$ ppm). Thin Layer Chromatography (TLC), Column chromatography (CC), Preparative Thin Layer Chromatography (PTLC), Solid Phase Extraction (SPE) and finally High Performance Liquid Chromatography (HPLC) was carried out to purify the antibacterial active ingredient in methanol crude extract. Secondary phytochemical screening was done using TLC spray reagents to identify the antibacterial active chemical compound class. Structure elucidation of isolated compound was carried out using Gas Chromatography Mass Spectrometry (GCMS) and Fourier-Transform InfraRed spectroscopy (FTIR). Two solvent systems, Ethyl acetate: methanol: deionized water (100:13.5:10) and Toluene, ethyl acetate (93:7) were optimised to separate and purify the active compounds in the crude extract by thin layer chromatography plates. One clear band with antibacterial active compounds was detected on the TLC plates, which were developed using Toluene, ethyl acetate (93:7) solvent system by bioautochromatography test and that band was named as A1 (Rf $=0.108)$. A1 showed antibacterial activity against Bacillus sp, E. coli and S.aureus. Three clear bands with antibacterial active compounds were detected on the TLC plates, which were developed using Ethyl acetate: Methanol: Water (100:13.5:10) solvent system by bioautochromatography test and that bands were named as $\mathrm{B} 1(\mathrm{Rf}=0.361), \mathrm{B} 2(\mathrm{Rf}=0.326), \mathrm{B} 3(\mathrm{Rf}=0.035) . \mathrm{B} 1$ showed antibacterial activity against Bacillus sp, E. coli and $S$. aureus. B2 showed antibacterial activity against E. coli and Bacillus sp. B3 showed antibacterial activity against Bacillus sp, E. coli and S.aureus. The three main fractions namely fraction 1, 2, 3 were obtained by PTLC and antibacterial activity of each fraction was verified using bioautochromatography and fraction 1 showed potential antibacterial activity whereas fraction 2 and 3 were not shown any activity against test organisms. Secondary chemical screening was given positive result for Arbutin Anthraquinone glycoside and Alkaloid. According to the GC-MS analysis was revealed that fraction 1 contain 3,5-bis (1-1-dimethylethyl)-4-hydroxy-octadecyl ester, 1- Hexadecene and 1-Heptadecanol. These results of the present study revealed that the cyanobacterium Lyngbya sp. had antibacterial activity against the tested human pathogenic bacteria and the Lyngbya sp. is a potential candidate to utilize as a natural source to produce antibiotics.
\end{abstract}

Keywords: Antibacterial activity, PTLC, TLC, HPLC

Proceedings of the $22^{\text {nd }}$ International Forestry and Environment Symposium 2017 of the Department of Forestry and Environmental Science, University of Sri Jayewardenepura, Sri Lanka 